\title{
Introduction to the Feature Section on Optical MEMS and Nanophotonics
}

$\mathbf{T}$ HE idea of fabricating tiny movable devices on chip was first conceived in the late 1960s, and strong research and development activity in this field started at around 1980. Since then, new materials, fabrication process, actuation mechanisms, and sensing methods have been proposed and demonstrated in a rapid-fire stream. The theoretical modeling of these developments has also advanced at a similar rate. In addition to device-level progress, the art of integration via microelectromechanical systems (MEMS) technology has itself advanced swiftly, yielding a huge array of integrated microsystems with rich and versatile functionality. The devices, which are inherently small, have already had significant impact on sensing, display, telecommunications, biology and medicine, and has contributed substantially to various applications in defense and security. Recently, the emergence of nanotechnology and nanofabrication naturally led to incorporation of nanoscale structures into sensing and actuation systems-NEMS, which opens yet another canvas for innovation in device and system technologies as well as their applications.

Optical MEMS emerged as a self-standing discipline of MEMS, after a great variety of integrated micro-optical components and sub-systems were first demonstrated using MEMS technology. As nanotechnology is combined with MEMS/NEMS technology, the emergence of nanophotonics has injected new research trends and dynamics into this field. This feature section is a follow-up to the 2009 IEEE International Conference on Optical MEMS and Nanophotonics. The 11 accepted papers give us a glimpse on some of the recent development in optical MEMS/NEMS technologies, the emerging field of nanophotonics and its application in optical micro- and nano-systems for sensing and actuations. The papers discuss challenging problems, such as optics for very short wavelengths (X-ray), large actuation, fine resolution tuning, high-speed reconfiguration, and tunable laser sources.

We are grateful to the authors who contributed to this issue, to the reviewers for their efforts ensuring the quality and to the staff of the IEEE/Photonics Society, who gave us so much support.
Lih Y. Lin, Guest Editor

Department of Electrical Engineering

University of Washington

Seattle, WA 98119-1997 USA

Hans Peter Herzig, Guest Editor

École Polytechnique Fédérale de Lausanne (EPFL) Neuchâtel 2000, Switzerland 


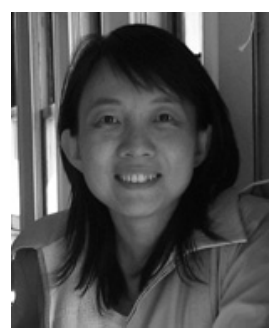

Lih Y. Lin (S'93-M'96-SM'02-F'10) received the Ph.D. degree in electrical engineering from University of California Los Angeles, Los Angeles, CA, in 1996.

She was with AT\&T Labs-Research as a Senior Technical Staff Member, and with Tellium, Inc. as the Director of Optical Technologies in 1996 and 2000, respectively. Since 2003, she has been with the Department of Electrical Engineering, University of Washington, Seattle, where she is currently a Professor. She has 54 journal papers and 130 conference publications. She holds 22 U.S. patents.

Dr. Lin received the MIT Technology Review 100 Award in 2003. She has served on the technical program committee and as the Chair and Co-Chair of various technical conferences, including the International Conference on Optical MEMS and Nanophotonics, the Optical Fiber Communications Conference, CLEO Pacific Rim, IEEE LEOS's Annual Meeting, OSA's Annual Meeting, and the OSA Photonics in Switching Topical Meeting. She was the Guest Editor for the Journal of Selected Topics in Quantum Electronics: Special Issue on Optical Micro- and Nanosystems and Journal of Lightwave Technology: Special Issue on Optical MEMS and its Future Trends. Currently, she is on the steering committee of the International Conference on Optical MEMS and Nanophotonics. She is a member of OSA.

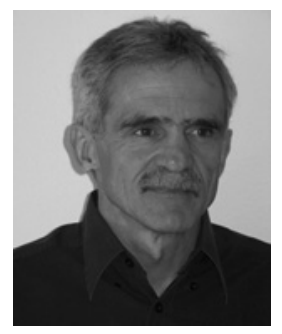

Hans Peter Herzig received the Diploma degree in physics from the Swiss Federal Institute of Technology, Zürich, Switzerland, in 1978, and the Ph.D. degree in optics from the University of Neuchâtel, Neuchâtel, Switzerland, in 1987.

From 1978 to 1982, he was a Scientist with the Department of Optics Development of Kern, Aarau, Switzerland, working in lens design and optical testing. In 1983, he was a Graduate Research Assistant with the Applied Optics Group, Institute of Microtechnology, University of Neuchâtel, Switzerland, working in the field of holographic optical elements. From 1989 to 2001, he was the Head of the Micro-Optics Research Group. From 2002 to 2008, he was a Full Professor and the Head of the Applied Optics Laboratory, University of Neuchâtel. He joined the faculty with the École Polytechnique Fédérale de Lausanne (EPFL), Switzerland, in 2009. Currently, he is a Professor with the EPFL and the President of the European Optical Society (EOS). His current research interests include refractive and diffractive micro-optics, nano-scale optics, and optical MEMS.

Dr. Herzig is a member of the OSA, IEEE Photonics Society, EOS, and SSOM. He served as the Conference Chairman for international conferences of EOS, IEE, IEEE/LEOS, OSA, and SPIE, and as a Guest Editor of three special issues of IEEE and OSA journals. He is the Editor of a well-known book on micro-optics (published in English and Chinese), author of 12 book chapters, 110 journal papers and 240 conference proceedings. 